\title{
PILOT AIDED SCHEDULING FOR UPLINK OFDMA
}

\author{
J.C.Vijayshree ${ }^{1}$, T.G. Palanivelu ${ }^{2}$ \\ ${ }^{1}$ Ph. D Research Scholar, Department of ECE, SCSVMV University, Kancheepuram - 631561 \\ ${ }^{2}$ Professor \& Head, Department of ECE, Sri Manakula Vinayagar Engineering College, Puducherry - 605107
}

\begin{abstract}
In the field of wireless communication, Energy efficiency is very important because of the expectation with wireless links to meet the increasing QoS demand. This results in increase of device power consumption. So we present an energy efficient scheduling using water filling algorithm with OFDMA technique for multiuser. Subcarrier allocation for a multiple access OFDM system is found out by this. Total bit rate is maximized by this approach. After the establishment of sub carrier allocation, the bit and power allocation for each user can be determined with a single-user. An energy efficient scheme based on time-averaged bits-per-joule metrics has been developed with low complexity. Simulation results proved the improvement in energy efficiency of the channel.
\end{abstract}

Keywords:- OFDMA, QoS, Subcarrier, Energy efficiency

\section{INTRODUCTION}

Tremendous growth has been experienced in wireless communication systems over the last few decades. This increasing quality of service requirements has to be met by limited network resources. It also results in increasing device power consumption. In this paper, a "bits-per-Joule" metric is used to target energy-efficiency. Several energy savings schemes already existed for link adaptation with optimal energy and resource allocation. With our approach, we tried to improve throughput with the optimization of power consumption.

Due to fairness issue, it is not possible to allocate all sub channels to a single user in a multi user system.[3] So, a system approach is most important for predicting the overall network energy efficiency. We have tried our investigation with the inclusion and exclusion of fairness constraint for uplink OFDMA systems with the assumption of channels as flat fading. This paper prove that the energy efficient power optimization approach improves energy as well as spectral efficiency and also reduces inter cell interferences. The link adaptations as well as resource allocation methods are designed with reduction in complexity and improvement in energy efficiency.

\section{SYSTEM OVERVIEW}

In this study, we consider the uplink OFDM multi-user multi-cell system. In each cell, a number of users can transmit simultaneously. Because of interference, we cannot rely on channel reciprocity.[1] The base station is responsible for resource allocation. Base station has to know the channel condition of users first, which can be done by finding the symbols or data sent by the intended user. At time instant $t_{1}$, base station collects the channel state information (CSI) from the users and at time instant $t_{2}$, the base station makes the scheduling decision. Following this decision, users start their transmission at time instant $t_{3}$. For RRM to work efficiently there is an underlying assumption:

$$
\operatorname{CSI}\left(t_{3}\right) \approx \operatorname{CSI}\left(t_{1}\right), \text { or say, } \operatorname{CSI}(t)=\operatorname{CSI}(t-\tau), \tau=t_{3}-t_{1}
$$

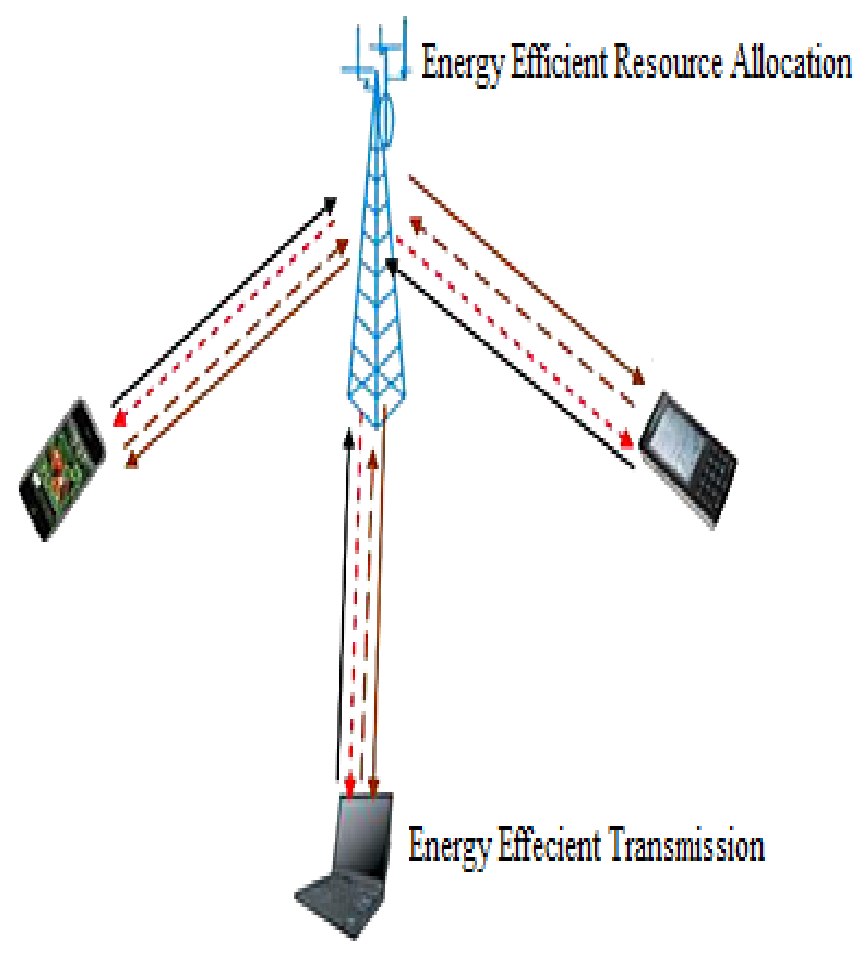

Fig 1 Uplink CSI delay

\subsection{CSI Metric}

In our study, the most common CSI metric, the SINR is used for analysis. The short term CSI gives an instantaneous value and long term CSI indicates higher order moments. In this paper, we calculated the short term CSI by measuring the SINR portion, with feedback delay.[9] 


$$
C S I_{i, n}(t)=\operatorname{SINR}_{i, n}(t-\tau)=P_{i, n}(t-\tau) \frac{\left|h_{i, n}(t-\tau)\right|^{2}}{I_{n}(t-\tau)+N_{n}}
$$

$C S I_{i, n}$ - CSI value of user $i$ in chunk $n$

$P_{i, n}$ - Power allocated to user $i$ on chunk $n$ at time $t-\tau$.

$h_{i, n}$ - Channel coefficient

$I_{n}+N_{n}$ - Interference and noise power in chunk $n, N_{n}$ is assumed constant.

\subsection{Power Control}

The transmission power for uplink is set using the formula

Power $=\min \left\{\mathrm{P}_{\text {max }}, 10 \log _{10}(\mathrm{RBs})+\mathrm{P}_{0}+\alpha \cdot \mathrm{PL}+\Delta_{\mathrm{tf}}+\mathrm{f}(\mathrm{i})\right\}$ $[\mathrm{dBm}][4]$

where

- $P_{\max }$ is the UE maximum power

- $10 \log _{10}(\mathrm{RBs})$ is the RB allocation

- $\mathrm{P}_{0}$ is the assumed interference

- $\alpha \cdot P L$ is the impact of path loss

- $\Delta_{\mathrm{tf}}$ is the MCS specific impact

- $\mathrm{f}(\mathrm{i})$ is the closed loop power control

\subsubsection{Bandwidth Dependent Power Control}

In this, we have not applied any path loss compensation and we assume constant power in each sub channel and the total power allocated to every user is purely proportional to the number of sub channels allotted.[10]

\subsubsection{Fixed Power Control}

Here, each user is provided with full path loss compensation and so it requires more power for transmission. So, with this method, for more path loss, even a single sub channel can be allocated for a user.[10]

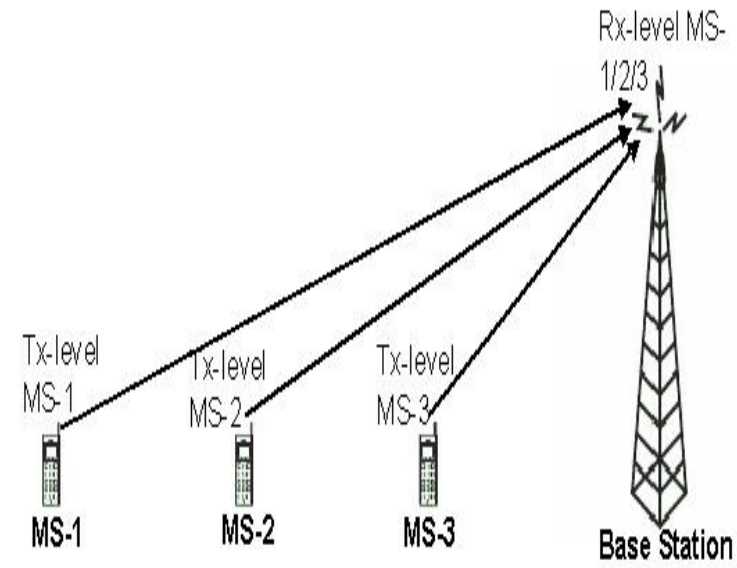

Without Power Control:

Tx level MS-1 = Tx level MS.2 = Tx level MS.3 $\longrightarrow$

Rx level MS-1 $<$ Rx level MS-2 $<$ Rx level MS-3

\section{With Power Control: \\ Txlevel MS.1 $>$ Tx level MS.2 $>$ Tx level MS.3 $\longrightarrow$ \\ Rx level MS-1 = Rx level MS-2 = Rx level MS-3}

Fig 2 Uplink with Power Control

\subsection{Link Adaptation}

The transmission parameters are adapted to the channel characteristics with the help of link adaptation. We use the fast link adaptation, in which the transmission parameters are adjusted in a frame basis. Link adaptation is done to improve the spectral efficiency. In uplink, more number of users may be scheduled in a frame, but the sub channels of a particular user are made to form a block, called the modulation and coding selection unit. The block is selected for every user in such a way that the number of information bits are maximised.

\subsubsection{Ideal Link Adaptation}

In ideal link adaptation, the SINR decides the user bit rate. Since, the SINR will be different with different sub channels of a multi carrier OFDM system, the effective SINR value has to be calculated taking in to account the differences. The values of SINR metric without the error background will be taken for the SINR calculation. [4]

\subsubsection{Non Ideal Link Adaptation}

In this case, the CSI is provided as the input to the link adaptation algorithm. If CSI reaches the real SINR value, then the transmission mode attained will be a proper one. In this approach, since there is no inter cell interference, we can optimize the SINR value of users. The accuracy will be high, due to the null interference variation with time.[4] 


\section{RESOURCE ALLOCATION}

Here we investigate the uplink resource allocation problem, with the constraint on the subcarrier allocation and the power limit of each user's equipment. Since e very user is independent and as we cannot coordinate the signals sent by them, we need a system for subcarrier allocation in order to ensure reliable transmission. We allocate the sub carrier power in such a way that it should be more than the minimum power required to carry the data in that channel and to overcome the Gaussian noise.[5]

\subsection{Simulation Environment}

Our studies are based on simulation results generated from a system level simulator, and there are some basic assumptions.

1. Uniform User distribution: Users are uniformly distributed in each cell. When modeling single cell system, the cell load is the exact number of users in the cell. While studying multi-cell system, cell load is the average number of users in each cell[1]

2. Full buffer Traffic mode: We analyze the traffic mode that allows every user to transmit at all times and we neglect the simulation time.[3]

3. Uplink FDD Duplex scheme: Since uplink is more important than the downlink, as it is the initiation of the communication process, which is facing more interference, we study the performance of uplink in frequency division duplexing mode.[7]

4. Single Input Single Output (SISO) Antenna System: Since the uplink process is started with mobile equipment, we consider the antenna system in our analysis as the single input single output antenna system.[1]

Table 1 lists some important parameters set for simulations. In the analysis of pilot aided scheduling for uplink OFDMA, we consider a friendly environment, which is a lower user speed environment.[7] In this case, the wireless channel varies slowly. With this slowly varying channel, the channel fading variation in time, the fading amplitude accounts for path loss, slow fading(shadow fading) and fast fading (Rayleigh fading) are smooth due to the low user speed (1 $\mathrm{m} / \mathrm{s}$ ). The noise power is assumed constant.

Table 1 Simulation Parameters [8]

\begin{tabular}{|l|l|}
\hline Central frequency & $2 \mathrm{GHz}$ \\
\hline Total bandwidth & $30 \mathrm{MHz}$ \\
\hline Sub-carrier bandwidth & $15 \mathrm{KHz}$ \\
\hline Number of subcarrier & 2000 \\
\hline Frame duration & $1 \mathrm{~ms}$ \\
\hline Traffic mode & full buffer \\
\hline Cell load & $5,10,20$ users/cell \\
\hline User speed & $1 \mathrm{~m} / \mathrm{s}$ \\
\hline Base station per site & 1 \\
\hline Cell radius & $500 \mathrm{~m}$ \\
\hline Number of cells & $1,7($ multi-cell) \\
\hline
\end{tabular}

\begin{tabular}{|l|l|}
\hline Noise power & $-159 \mathrm{dBm}$ \\
\hline Maximum Mobile Power & $1 \mathrm{w}$ \\
\hline CSI feedback delay & $2 \mathrm{~ms}$ \\
\hline Persistent scheduling interval & $10 \mathrm{~ms}$ \\
\hline HARQ mode & incremental redundancy \\
\hline
\end{tabular}

\section{SIMULATION RESULTS AND DISCUSSION}

The performance with energy efficiency for uplink model was simulated. In this, we first obtain the channel energy efficiency by varying the filter coefficient. Filter coefficient here means the power allocation in each sub channel. The power allocation is designed by MMSE and LSE methods with including pilot power. Also, we analyzed the capacity of bit rate using these.

\subsection{Performance of SNR}

The simulation result of signal to noise ratio analysis is shown in Figure 3. It is inferred from the graph that the signal to noise ratio is high when the bit error rate is less than $10^{-6}$. We analyzed this for finding the signal power at desired rate, since the signal to noise ratio power must be lesser than the transmitting power in order to avoid the noises occurs in the signal.

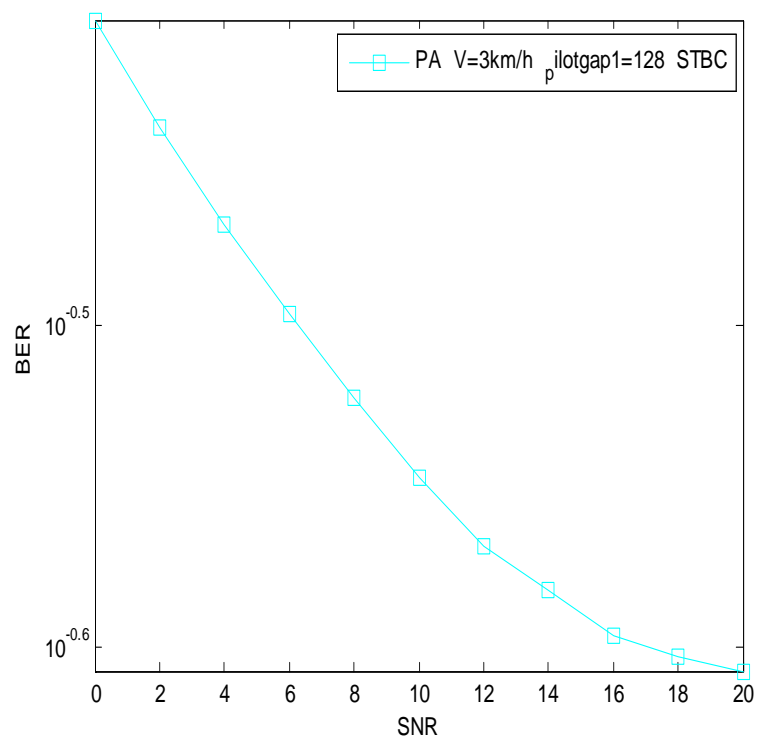

Fig 3 Performance of BER vs. SNR

\subsection{Capacity of Bit Rate}

The simulation result of bit rate capacity analysis is shown in Figure 4. The graph shows that the capacity of bit rate through the channel by means of iterative algorithm. Also we estimate the bit rate with Arithmetic Mean, it obtained by using MMSE and LES methods with pilot power. 


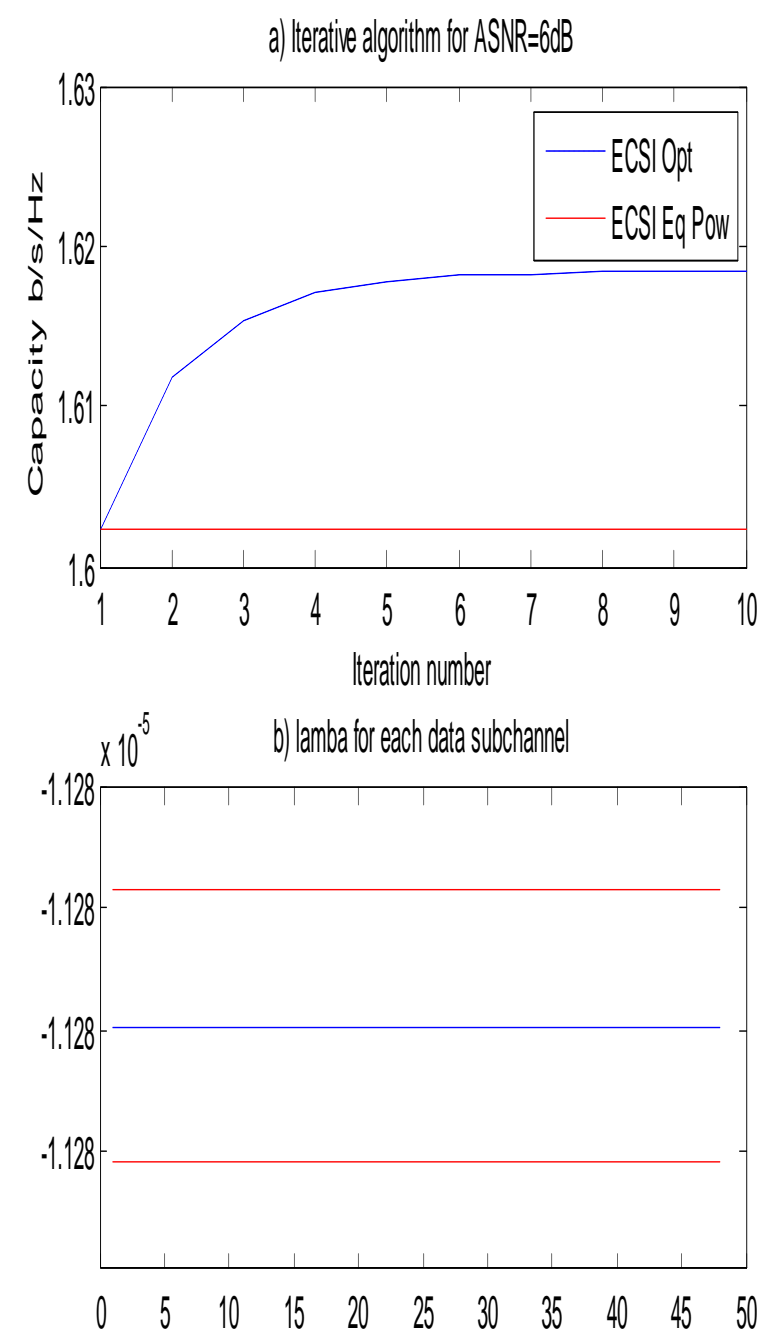

Fig 4 Bit Rate Capacity Analysis

\subsection{Power Estimation of Channels}

The simulation result over power estimation of channel is shown in Figure 5. The first graph shows the amount of power allocated to each sub channel and the estimated error in those channels. The second graph shows the cumulative power of the channels based on the usage.
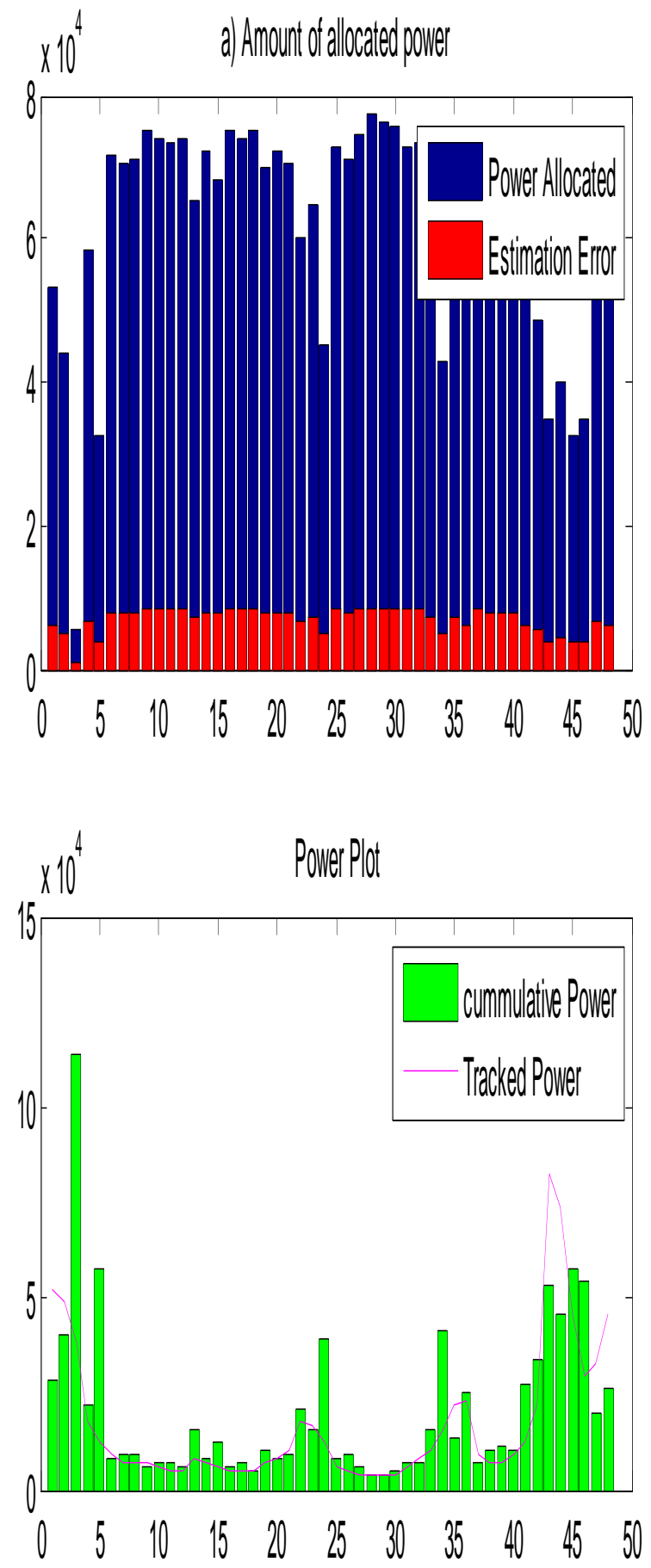

Fig 5 Power estimation of channels

\subsection{Energy Efficiency of Channel}

The simulation result for energy efficiency of channel is shown in Figure 6. With the variation of filter coefficient, we estimate the energy efficiency of channel by mean of Arithmetic Mean. 


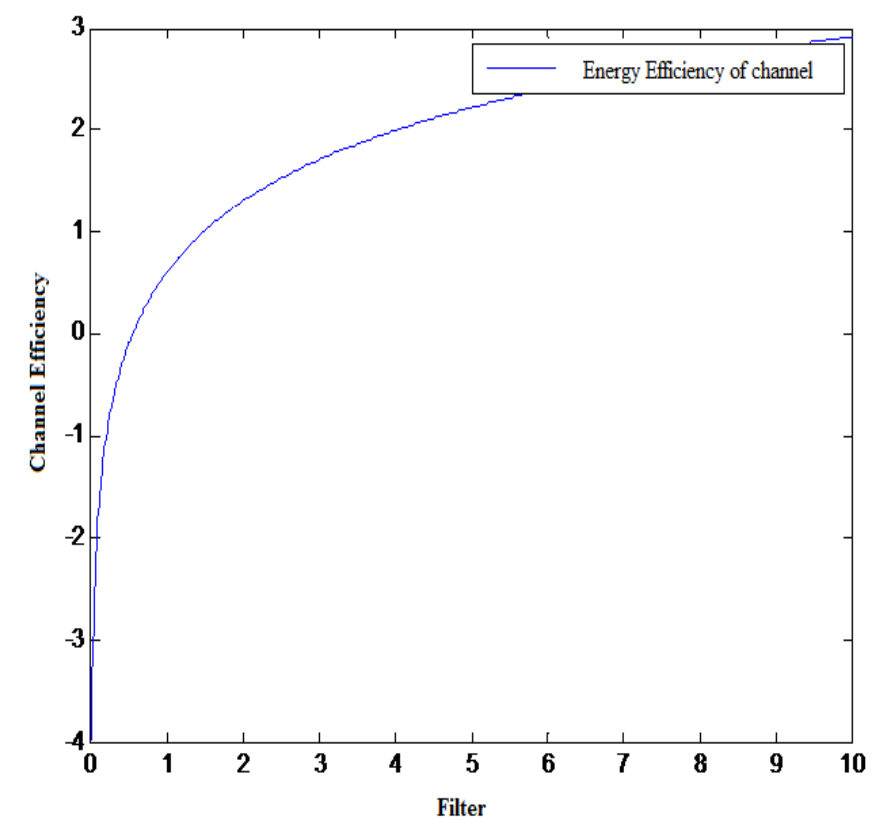

Fig 6 Energy efficiency of channel

The simulation result of energy efficiency for uplink model was obtained by analyzing the bit error performance to obtain the maximum throughput with varying noise power and we design the channel coefficient power for estimating the channel efficiency. Pilot power is included in the filter weight to avoid the inter carrier interference. In addition to this, we also analyze capacity of bit rate through the channel with different power allocation techniques. They are 1 . Adaptive power allocation through water filling algorithm with AM, 2. Equal power allocation with Arithmetic Mean and 3. Maximum Iterative Capacity with AM metrics. We design the sub channels for the multiuser access as one channel at a time. It is achieved by using OFDMA. In our simulation result, we got the maximum bit rate in the adaptive power allocation through water filling algorithm with AM techniques. In this we also present the energy efficiency of channel.

\section{CONCLUSIONS}

As mobile stations cannot be permanently powered, We have considered uplink energy-efficient communications in OFDMA systems. In this, we developed the energy efficient pilot aided scheduling method for uplink OFDMA communication system. With focus on optimizing time average energy efficiency metric, a closed form link adaptation scheme is obtained at the beginning for frequency selective channels. In our paper the energy efficiency has been analyzed by means of water filling algorithm by considering the maximum achievable capacity given pilot position and power, Capacity with equal power allocation for data sub channels based on MMSE channel estimation and LSE channel estimation. Also we analyzed the capacity of bit rate through the channel estimations and we produce the bit error performance by the variation in the signal to noise ratio.

\section{REFERENCES}

[1] Guowang Miao, Nageen Himayat, Geoffrey Ye Li, and Shilpa Talwar, "Low Complexity EnergyEfficient Scheduling for Uplink OFDMA" IEEE Transaction on communication vol. 60, No. 1, January 2012.

[2] K. Lahiri, A. Raghunathan, S. Dey, and D. Panigrahi, "Battery-driven System design: a new frontier in low power design," in Proc. 2002 Intl.Conf. VLSI Design, pp. 261-267.

[3] G. W. Miao, N. Himayat, Y. Li, and A. Swami, "Cross-layer optimization for energy-efficient wireless communications: a survey," vol. 9, no. 4, pp. 529-542, Apr. 2009.

[4] R. G. Gal lager, "Power limited channels: coding, multi-access, and spread Spectrum," in Proc. 1988 Conf. Inf. Sci. Syst., vol. 1.

[5] G. Song and Y. Li, "Adaptive subcarrier and power allocation In OFDM based on maximizing utility," in Proceedings' of the 57th IEEE Semiannual Vehicular Technology Conference (VTC)

[6] vol. 2, pp. 905 Z. Shen, J. G. Andrews, and B. L. Evans, "Optimal power Allocation in multiuser OFDM systems," in Proceedings of The IEEE Global Telecommunications Conference (GLOBECOM)

[7] G. W. Miao, N. Himayat, Y. Li, and D. Bormann, "Energy-efficient Design in wireless OFDMA," in Proc. IEEE ICC 2008, May 2008, pp. 3307-3312.

[8] G. Miao, N. Himayat, and Y. Li, "Energy-efficient transmission in Frequency-selective channels," in Proc. IEEE Globecom 2008, Nov. 2008, pp. 1-5.

[9] S. Cui, A. J. Goldsmith, and A. Bahai, "Energyconstrained modulation Optimization," IEEE Trans. Wireless Communication vol. 4, no. 5, pp. 23492360, Sep. 2005.

[10] Z. Xu, C. Li, G. and Yang, S. Zhang, Y. Chen, and S. $\mathrm{Xu}$, "Energy efficient Power allocation for pilots in training based downlink OFDMA Systems," IEEE Trans. On Communication, Apr 2011 